\title{
Cine acquisition strategies for visualizing atrial septal defects by CMR
}

\author{
Arun Natarajan*, Evangelia Nyktari, Arun J Baksi, Heiko E Kindler, Philip J Kilner \\ From 16th Annual SCMR Scientific Sessions \\ San Francisco, CA, USA. 31 January - 3 February 2013
}

\section{Background}

Atrial septal defects (ASDs) may escape detection before adulthood, particularly those in unusual locations, which may elude echocardiographic visualisation. CMR is established for the quantification of shunt flow and, if appropriately acquired, can provide clear visualisation of ASDs and adjacent structures to inform decisions regarding intervention. Our objective is to recommend cardiovascular magnetic resonance (CMR) cine acquisition strategies suitable for the visualisation of the less common as well as the common types of ASD.

\section{Methods}

In the CMR Unit of a tertiary referral centre for adults with congenital heart disease we retrospectively reviewed the CMRs of patients with unoperated ASDs over a 3 year period to assess the suitability of cine acquisition strategies for the visualisation of different types of ASD and any associated anomalies of pulmonary venous connection.

\section{Results}

157 patients with unoperated ASDs had CMR studies in 3 years. If already suspected, we had routinely acquired an 'atrial stack' of cines, meaning a contiguous stack, $5 \mathrm{~mm}$ thick, parallel to the routine ventricular short axis (SA) stack, stepping backward from the basal ventricular plane until the superior vena cava was identifiable. This orientation visualized ostium secundum ASDs well $(n=117)$, and was also good for inferior sinus venosus defects $(n=2)$ and unroofed coronary sinus $(n=3)$. In superior sinus venosus defects $(n=21)$, a transaxial cine stack was found to give clearer visualisation of both the defect and any associated anomalous pulmonary vein connection(s) $(n=20$, plus 4 anomalous connections identified with secundum ASDs). The transaxial cine orientation was also the more suitable one for atrio-ventricular septal defects (AVSD, $n=15$ ) as it depicted insertions of the $\mathrm{A}-\mathrm{V}$ valve leaflets adjacent to the $\operatorname{defect}(\mathrm{s})$.

\section{Conclusions}

In patients with suspected ASDs, we recommend the acquisition of an atrial SA cine stack and a transaxial cine stack that covers atrial to aortic arch levels. Additional oblique cines aligned with the defect can supplement these, as indicated in the table.

\section{Funding}

None.

Table 1 Cine orientations for visualizing atrial septal defects and anomalous pulmonary veins.

\begin{tabular}{cccc}
\hline & Atrial short axis stack & Transaxial stack & Additional oblique planes \\
\hline Ostium secundum defect & Good & Satisfactory & 4-chamber cine \\
Superior sinus venosus defect & Satisfactory & Good & 3-chamber cine \\
Atrioventricular septal defect & Suboptimal & Good & 4-chamber cine \\
Unroofed coronary sinus & Good & Suboptimal & Consider whether the coronary sinus roof is visible in all long axis cines \\
Inferior sinus venosus defect & Good & Suboptimal & Oblique sagittal cine \\
Anomalous pulmonary veins & Suboptimal & Good & Oblique coronal cine \\
\hline
\end{tabular}

CMR Unit, Royal Brompton Hospital, London, UK, London, U K 
- Convenient online submission

- Thorough peer review

- No space constraints or color figure charges

- Immediate publication on acceptance

- Inclusion in PubMed, CAS, Scopus and Google Scholar

- Research which is freely available for redistribution 